DOI 10.1007/s11253-019-01599-7

Ukrainian Mathematical Journal, Vol.70, No.11, April, 2019 (Ukrainian Original Vol.70, No.11, November, 2018)

O. M. Atlasiuk, V. A. Mikhailets (Institute of Mathematics of NAS of Ukraine, Kyiv)

\title{
Fredholm one-dimensional boundary-value problems with parameter in Sobolev spaces
}

\begin{abstract}
For systems of linear differential equations on a compact interval, we investigate the dependence on a parameter $\varepsilon$ of the solutions to boundary-value problems in the Sobolev spaces $W_{\infty}^{n}$. We obtain a constructive criterion of the continuous dependence of the solutions of these problems on the parameter $\varepsilon$ for $\varepsilon=0$. The degree of convergence of these solutions is established.
\end{abstract}

\section{Introduction}

The investigation of solutions of the systems of ordinary differential equations is an important part of numerous problems of contemporary analysis and its applications (see, e.g., [1] and the references therein). For linear boundary-value problems, the conditions for the Fredholm property and continuous dependence of solutions on the parameters were established by Kiguradze [2,3]. Later, his results were generalized by the second author of the present paper and his colleagues [4-6]. Recently, these investigations were extended to more general classes of Fredholm boundary-value problems in various Banach function spaces [7-11]. These problems have a series of specific features and require the application of new approaches and methods.

\section{Statement of the problem}

Consider a finite interval $(a, b) \subset \mathbb{R}$ and given numbers

$$
\{m, n\} \subset \mathbb{N}, \quad \varepsilon_{0}>0 .
$$

We study a family of inhomogeneous boundary-value problems of the form

$$
\begin{gathered}
L(\varepsilon) y(t ; \varepsilon):=y^{\prime}(t ; \varepsilon)+A(t ; \varepsilon) y(t ; \varepsilon)=f(t ; \varepsilon), \quad t \in(a, b), \\
B(\varepsilon) y(\cdot ; \varepsilon)=c(\varepsilon),
\end{gathered}
$$

parametrized by a number $\varepsilon \in\left[0, \varepsilon_{0}\right)$. Here, for any fixed value of the parameter $\varepsilon$, the matrix function

$$
A(\cdot ; \varepsilon) \in W_{\infty}^{n-1}\left([a, b] ; \mathbb{C}^{m \times m}\right)=:\left(W_{\infty}^{n-1}\right)^{m \times m}
$$

the vector function

$$
f(\cdot ; \varepsilon) \in W_{\infty}^{n-1}\left([a, b] ; \mathbb{C}^{m}\right)=:\left(W_{\infty}^{n-1}\right)^{m}
$$

the vector $c(\varepsilon) \in \mathbb{C}^{m}$, and $B(\varepsilon)$ is a linear continuous operator

$$
B(\varepsilon):\left(W_{\infty}^{n}\right)^{m} \rightarrow \mathbb{C}^{m} .
$$


A solution of the boundary-value problem (11), (2) is defined as a vector function $y(\cdot ; \varepsilon) \in$ $\left(W_{\infty}^{n}\right)^{m}$ satisfying equation (1) almost everywhere on $(a, b)$ (everywhere for $n \geq 1$ ) and equality (2). The boundary condition (2) is the most general condition for system (11) whose solution runs over the entire Sobolev space $\left(W_{\infty}^{n}\right)^{m}$ [11, Lemma 1]. The boundary-value problem (1), (2) can be associated with the linear operator

$$
(L(\varepsilon), B(\varepsilon)):\left(W_{\infty}^{n}\right)^{m} \rightarrow\left(W_{\infty}^{n-1}\right)^{m} \times \mathbb{C}^{m} .
$$

This is a Fredholm operator with index zero [11, Theorem 1].

The main aim of the present paper is to establish a criterion for the continuous dependence of the solutions of boundary-value problems of the form (11), (2) on the parameter $\varepsilon$ for $\varepsilon=0$.

\section{Main results}

We now formulate the main results of the present paper. Their proof is presented in Section 4 .

In order that the analyzed problem be meaningful, in what follows, we assume than condition (0) is satisfied, namely, a boundary-value problem of the form (11), (2)

$$
L(0) y(t ; 0)=0, \quad t \in(a, b), \quad B(0) y(\cdot ; 0)=0
$$

possesses solely the trivial solution.

In this case, the corresponding limiting inhomogeneous boundary-value problem possesses a unique solution.

Consider the following boundary conditions as $\varepsilon \rightarrow 0+$ :

(I) $A(\cdot ; \varepsilon) \rightarrow A(\cdot ; 0)$ in the space $\left(W_{\infty}^{n-1}\right)^{m \times m}$;

(II) $B(\varepsilon) y \rightarrow B(0) y$ in $\mathbb{C}^{m}$ for any $y \in\left(W_{\infty}^{n}\right)^{m}$.

Definition 1. We say that a solution of the boundary-value problem (11), (2) continuously depends on the parameter $\varepsilon$ for $\varepsilon=0$ if the following conditions are satisfied:

(*) there exists a positive number $\varepsilon_{1}<\varepsilon_{0}$ such that, for any $\varepsilon \in\left[0, \varepsilon_{1}\right)$, arbitrary right-hand sides $f(\cdot ; \varepsilon) \in\left(W_{\infty}^{n-1}\right)^{m}$, and $c(\varepsilon) \in \mathbb{C}^{m}$, this problem has a unique solution $y(\cdot ; \varepsilon)$ that belongs to the space $\left(W_{\infty}^{n}\right)^{m}$;

$(* *)$ the convergence of the right-hand sides $f(\cdot ; \varepsilon) \rightarrow f(\cdot ; 0)$ in $\left(W_{\infty}^{n-1}\right)^{m}$ and $c(\varepsilon) \rightarrow c(0)$ in $\mathbb{C}^{m}$ implies the convergence of the solutions

$$
y(\cdot ; \varepsilon) \rightarrow y(\cdot ; 0) \quad \text { in } \quad\left(W_{\infty}^{n}\right)^{m} \quad \text { as } \quad \varepsilon \rightarrow 0+.
$$

We now formulate a criterion for the continuity of the solution $y=y(t, \varepsilon)$ of the boundaryvalue problem (11), (2) with respect to the parameter $\varepsilon$ as $\varepsilon \rightarrow 0+$ in the space $W_{\infty}^{n}$.

Theorem 1. A solution of the boundary-value problem (11), (2) continuously depends on the parameter $\varepsilon$ for $\varepsilon=0$ if and only if it satisfies condition (0) and the boundary conditions (I) and (II). 
We proceed to the investigation of the rate of convergence of solutions to the boundary-value problem (1), (2) as $\varepsilon \rightarrow 0+$.

We set

$$
\widetilde{d}_{n-1, \infty}(\varepsilon):=\|L(\varepsilon) y(\cdot ; 0)-f(\cdot ; \varepsilon)\|_{n-1, \infty}+\|B(\varepsilon) y(\cdot ; 0)-c(\varepsilon)\|_{\mathbb{C}^{m}}
$$

where $\|\cdot\|_{n-1, \infty}$ is the norm in the space $W_{\infty}^{n-1}$ and $\|\cdot\|_{\mathbb{C}^{m}}$ is the norm in the space $\mathbb{C}^{m}$.

The quantities

$$
\|y(\cdot ; 0)-y(\cdot ; \varepsilon)\|_{n, \infty}
$$

and $\widetilde{d}_{n-1, \infty}(\varepsilon)$ are, respectively, the error and discrepancy of the solution $y(\cdot ; \varepsilon)$ of the boundaryvalue problem (11), (2) if $y(\cdot ; \varepsilon)$ is its exact solution and $y(\cdot ; 0)$ is an approximate solution of the problem.

Theorem 2. Suppose that the boundary-value problem (1), (2) satisfies conditions (0), (I), and (II). Then there exist positive quantities $\varepsilon_{2}<\varepsilon_{1}$ and $\gamma_{1}, \gamma_{2}$ such that, for any $\varepsilon \in\left(0, \varepsilon_{2}\right)$, the following two-sided estimate is true:

$$
\gamma_{1} \widetilde{d}_{n-1, \infty}(\varepsilon) \leq\|y(\cdot ; 0)-y(\cdot ; \varepsilon)\|_{n, \infty} \leq \gamma_{2} \widetilde{d}_{n-1, \infty}(\varepsilon),
$$

where the quantities $\varepsilon_{2}, \gamma_{1}$ and $\gamma_{2}$ are independent $y(\cdot ; 0)$ and $y(\cdot ; \varepsilon)$.

By virtue of this theorem, the error and discrepancy of the solution $y(\cdot ; \varepsilon)$ of the boundaryvalue problem (1), (2) have the same order of smallness.

\section{Auxiliary results}

The theorem presented below contains constructive conditions under which the continuous operator (3) is invertible for sufficiently small values of the parameter $\varepsilon$ and guarantees the continuous dependence of solutions on the parameter in the space $\left(W_{\infty}^{n}\right)^{m}$.

Theorem 3. Suppose that the following conditions are satisfied as $\varepsilon \rightarrow 0+$ :

1) $\|A(\cdot ; \varepsilon)-A(\cdot ; 0)\|_{n-1, \infty} \rightarrow 0$ in the space $\left(W_{\infty}^{n-1}\right)^{m \times m}$;

2) $B(\varepsilon) y \rightarrow B(0) y$ for any $y \in\left(W_{\infty}^{n}\right)^{m}$.

Then, for sufficiently small $\varepsilon>0$, the operator $(L(\varepsilon), B(\varepsilon))$ is invertible. In addition, if

3) $\|f(\cdot ; \varepsilon)-f(\cdot ; 0)\|_{n-1, \infty} \rightarrow 0$ and $c(\varepsilon) \rightarrow c(0)$,

then the solution $y(\cdot, \varepsilon)$ of problem (11), (2) satisfies the limit property

$$
\|y(\cdot ; \varepsilon)-y(\cdot ; 0)\|_{n, \infty} \rightarrow 0 .
$$

We present the proof of Theorem 3 in the form of four lemmas formulated in what follows:

Lemma 1. Suppose that condition (0) and conditions 1) and 2) of Theorem 3 are satisfied. Then, for sufficiently small $\varepsilon>0$, the operator $(L(\varepsilon), B(\varepsilon))$ is invertible. 
Proof. Under condition 1), by the theorem on homeomorphisms from [11], we get

$$
\|Y(\cdot ; \varepsilon)-Y(\cdot ; 0)\|_{n, \infty} \rightarrow 0, \quad \varepsilon \rightarrow 0+.
$$

Thus, by using condition 2), we establish the convergence of numerical matrices:

$$
[B(\varepsilon) Y(\cdot ; \varepsilon)] \rightarrow[B(0) Y(\cdot ; 0)], \quad \varepsilon \rightarrow 0+.
$$

According to condition (0), the limit square matrix is nonsingular [11, Theorem 2]. Hence, for sufficiently small $\varepsilon \geq 0$, we find

$$
\operatorname{det}[B(\varepsilon) Y(\cdot ; \varepsilon)] \neq 0 .
$$

This yields the invertibility of the operator $(L(\varepsilon), B(\varepsilon))$.

Parallel with the original inhomogeneous boundary-value problem (1), (2) for the vector function $y(t ; \varepsilon)$, we consider the following three vector boundary-value problems:

$$
\begin{gathered}
v^{\prime}(t ; \varepsilon)=-A(t ; \varepsilon) v(t ; \varepsilon), \quad B(\varepsilon) v(\cdot ; \varepsilon)=c(\varepsilon), \\
x^{\prime}(t ; \varepsilon)+A(t ; \varepsilon) x(t ; \varepsilon)=f(t ; \varepsilon), \quad x(a ; \varepsilon)=0, \\
w^{\prime}(t ; \varepsilon)+A(t ; \varepsilon) w(t ; \varepsilon)=f(t ; \varepsilon), \quad B(\varepsilon) w(\cdot ; \varepsilon)=0,
\end{gathered}
$$

where the parameter $\varepsilon \geq 0$ is small. It is known that the boundary-value (Cauchy) problem (9) is uniquely solvable.

By using Lemma 9, we arrive at the equality

$$
y(\cdot ; \varepsilon)=v(\cdot ; \varepsilon)+w(\cdot ; \varepsilon)
$$

for small $\varepsilon \geq 0$. Hence, in order to prove Theorem 3, it suffices to show that, under its conditions, the following relations hold as $\varepsilon \rightarrow 0+$ :

$$
\begin{gathered}
\|v(\cdot ; \varepsilon)-v(\cdot ; 0)\|_{n, \infty} \rightarrow 0 \\
\|w(\cdot ; \varepsilon)-w(\cdot ; 0)\|_{n, \infty} \rightarrow 0
\end{gathered}
$$

Lemma 2. Suppose that the conditions of Theorem 3 are satisfied as $\varepsilon \rightarrow 0+$. Then the limit relation (11) is true.

Proof. By using the first equality in the boundary-value problem (8), we obtain

$$
v(\cdot ; \varepsilon)=Y(\cdot ; \varepsilon) \widetilde{c}(\varepsilon)
$$

for some $\widetilde{c}(\varepsilon) \in \mathbb{C}^{m}$. In view of the second equality in problem (8), we find

$$
[B(\varepsilon) Y(\cdot ; \varepsilon)] \widetilde{c}(\varepsilon)=c(\varepsilon) .
$$

By virtue of Lemma 1, the criterion of invertibility from [11, Theorem 2], relation (7), and condition 2), we get

$$
\widetilde{c}(\varepsilon)=[B(\varepsilon) Y(\cdot ; \varepsilon)]^{-1} c(\varepsilon) \rightarrow[B(0) Y(\cdot ; 0)]^{-1} c(0)=\widetilde{c}(0), \quad \varepsilon \rightarrow 0+.
$$

Relation (11) is derived from (6) and (13) . 
Lemma 3. Suppose that conditions 1) 3) of Theorem 3 are satisfied as $\varepsilon \rightarrow 0+$ Then the solution of problem (9) has the following property:

$$
\|x(\cdot ; \varepsilon)-x(\cdot ; 0)\|_{n, \infty} \rightarrow 0, \quad \varepsilon \rightarrow 0+.
$$

Proof. Assume that the number $\varepsilon>0$ is sufficiently small. The solution of problem (9) admits the following representation:

$$
x(t ; \varepsilon)=Y^{-1}(t ; \varepsilon) \int_{a}^{t} Y(s ; \varepsilon) f(s ; \varepsilon) \mathrm{d} s .
$$

Under condition 1), by the theorem on homeomorphisms in [11], we get

$$
\left\|Y^{ \pm 1}(\cdot ; \varepsilon)-Y^{ \pm 1}(\cdot ; 0)\right\|_{n, \infty} \rightarrow 0
$$

as $\varepsilon \rightarrow 0+$. According to condition 3 ) and relation (16), we find

$$
\|Y(\cdot ; \varepsilon) f(\cdot ; \varepsilon)-Y(\cdot ; 0) f(\cdot ; 0)\|_{n-1, \infty} \rightarrow 0
$$

because $W_{\infty}^{n}$ is a Banach algebra. Thus, relation (14) follows from relations (15)-(17).

Lemma 4. Under the conditions of Theorem [3, the limit relation (12) is true.

Proof. The vector function $u(\cdot ; \varepsilon)=x(\cdot ; \varepsilon)-w(\cdot ; \varepsilon)$ is a solution of a boundary-value problem of the form (88):

$$
\begin{aligned}
u^{\prime}(t ; \varepsilon) & =-A(t ; \varepsilon) u(t ; \varepsilon), \\
B(\varepsilon) u(\cdot ; \varepsilon) & =B(\varepsilon) x(\cdot ; \varepsilon)=: \widetilde{c}(\varepsilon) .
\end{aligned}
$$

By using property 2) and Lemma [3, we get $\widetilde{c}(\varepsilon) \rightarrow \widetilde{c}(0)$ as $\varepsilon \rightarrow 0+$. It follows from Lemma 2 that

$$
\|u(\cdot ; \varepsilon)-u(\cdot ; 0)\|_{n, \infty} \rightarrow 0, \quad \varepsilon \rightarrow 0+.
$$

In view of the equality $w(\cdot ; \varepsilon)=x(\cdot ; \varepsilon)-u(\cdot ; \varepsilon)$ and relations (14) and (18), we obtain (12).

The required limit property (5) is a direct corollary of equality (10) and Lemmas 2 and 4 .

Theorem 3 is proved.

Remark 1. Definition 1 is equivalent to the following definition:

Definition 2. We say that a solution of the boundary-value problem (11), (2) continuously depends on the parameter $\varepsilon$ for $\varepsilon=0$ if the following conditions are satisfied:

(*) there exists a positive number $\varepsilon_{1}<\varepsilon_{0}$ such that, for any $\varepsilon \in\left[0, \varepsilon_{1}\right)$, arbitrary right-hand sides $f(\cdot) \in\left(W_{\infty}^{n-1}\right)^{m}$, and $c \in \mathbb{C}^{m}$, this problem possesses a unique solution $y(\cdot ; \varepsilon) \in$ $\left(W_{\infty}^{n}\right)^{m}$

$(* *)$ the following limit relation for the convergence of solutions is true:

$$
y(\cdot ; \varepsilon) \rightarrow y(\cdot ; 0) \quad \text { in } \quad\left(W_{\infty}^{n}\right)^{m} \quad \text { as } \quad \varepsilon \rightarrow 0+.
$$


The conditions of Definition 2 directly follow from Definition 1. We now prove the converse implication.

By Theorem 3, the operator $(L(\varepsilon), B(\varepsilon))$ has a bounded inverse operator

$$
(L(\varepsilon), B(\varepsilon))^{-1}:\left(W_{\infty}^{n-1}\right)^{m} \times \mathbb{C}^{m} \rightarrow\left(W_{\infty}^{n}\right)^{m}
$$

for any $\varepsilon \in\left[0, \varepsilon_{2}^{\prime}\right)$. Moreover, by Definition 2, for sufficiently small $\varepsilon$, we get the following strong convergence of inverse operators:

$$
(L(\varepsilon), B(\varepsilon))^{-1} \stackrel{s}{\rightarrow}(L(0), B(0))^{-1},
$$

as well as the convergence of right-hand sides:

$$
f(\cdot ; \varepsilon) \rightarrow f(\cdot ; 0), \quad c(\varepsilon) \rightarrow c(0) .
$$

We choose $f(\cdot ; \varepsilon) \in\left(W_{\infty}^{n-1}\right)^{m}$ and $c(\varepsilon) \in \mathbb{C}^{m}$. Then the equalities

$$
\begin{aligned}
& y(\cdot ; \varepsilon)=(L(\varepsilon), B(\varepsilon))^{-1}(f(\cdot ; \varepsilon), c(\varepsilon)), \\
& y(\cdot ; 0)=(L(0), B(0))^{-1}(f(\cdot ; 0), c(0)),
\end{aligned}
$$

are true, i.e., the convergence of $y(\cdot ; \varepsilon)$ to $y(\cdot ; 0)$ is equivalent to the convergence

$$
(L(\varepsilon), B(\varepsilon))^{-1}(f(\cdot ; \varepsilon), c(\varepsilon)) \rightarrow(L(0), B(0))^{-1}(f(\cdot ; \varepsilon), c(\varepsilon)), \quad \varepsilon \rightarrow 0+.
$$

By the Banach-Steinhaus theorem, for sufficiently small $\varepsilon$, we obtain

$$
\left\|(L(\varepsilon), B(\varepsilon))^{-1}\right\| \leqslant C .
$$

Since

$$
\begin{gathered}
\left\|(L(\varepsilon), B(\varepsilon))^{-1}(f(\cdot ; \varepsilon), c(\varepsilon))-(L(0), B(0))^{-1}(f(\cdot ; 0), c(0))\right\| \leqslant \\
\leqslant\left\|(L(\varepsilon), B(\varepsilon))^{-1}\right\|\|(f(\cdot ; \varepsilon), c(\varepsilon))-(f(\cdot ; 0), c(0))\|+ \\
\quad+\left\|\left[(L(\varepsilon), B(\varepsilon))^{-1}-(L(0), B(0))^{-1}\right](f(\cdot ; 0), c(0))\right\|,
\end{gathered}
$$

by using conditions (19)-(24), we prove that the limit relation $(* *)$ in Definition 1 is true.

We now establish one more auxiliary result. Assume that the operator $(L(0), B(0))$ is invertible. We now consider the following two conditions:

(i) the operator $(L(\varepsilon), B(\varepsilon))^{-1}$ converges to the operator $(L(0), B(0))^{-1}$ in the strong operator topology;

(ii) the operator $(L(\varepsilon), B(\varepsilon))$ converges to the operator $(L(0), B(0))$ in the strong operator topology.

Theorem 4. Conditions $(i)$ and (ii) are equivalent, i.e., as $\varepsilon \rightarrow 0+$,

$$
(L(\varepsilon), B(\varepsilon))^{-1} \stackrel{s}{\rightarrow}(L(0), B(0))^{-1} \Longleftrightarrow(L(\varepsilon), B(\varepsilon)) \stackrel{s}{\rightarrow}(L(0), B(0)) .
$$

We split the procedure of substantiation of equivalence (25) into two steps.

Step 1. We prove that the strong convergence of inverse operators is equivalent to the set of conditions (I) and (II).

Step 2. We prove that the set of conditions (I) and (II) is equivalent to the strong convergence of operators. 
Indeed, by Theorem 11, the operator $(L(\varepsilon), B(\varepsilon))$ has a bounded inverse operator $(L(\varepsilon), B(\varepsilon))^{-1}$. Moreover, we also have strong convergence of the inverse operators. Thus, Theorem 1 immediately implies the validity of conditions of Step 1. Note that, for any continuous operators acting in infinite-dimensional Banach spaces and depending on $\varepsilon$, this equivalence is not true.

We now proceed to Step 2. To prove this assertion, we show that the following lemma is true:

Lemma 5. The boundary condition (I) is equivalent to each of the following conditions:

$\left(a_{1}\right)\|L(\varepsilon)-L(0)\| \rightarrow 0$ as $\varepsilon \rightarrow 0+;$

$\left(a_{2}\right) L(\varepsilon) y \rightarrow L(0) y$ in $\left(W_{\infty}^{n-1}\right)^{m}$ as $\varepsilon \rightarrow 0+$ for every $y \in\left(W_{\infty}^{n}\right)^{m}$.

Proof. The implication $\left(a_{1}\right) \Rightarrow\left(a_{2}\right)$ is obvious. It remains to show that condition $\left(a_{1}\right)$ follows from the boundary condition (I) and the boundary condition (I) follows from condition $\left(a_{2}\right)$. We first substantiate the first implication. Assume that $\|A(\varepsilon)-A(0)\|_{n-1, \infty} \rightarrow 0$ as $\varepsilon \rightarrow 0+$. For any vector function $y \in\left(W_{\infty}^{n}\right)^{m}$, we obtain

$$
\begin{gathered}
\|(L(\varepsilon)-L(0)) y\|_{n-1, \infty}=\|(A(\varepsilon)-A(0)) y\|_{n-1, \infty} \leq \\
\leq c_{n-1, \infty}\|A(\varepsilon)-A(0)\|_{n-1, \infty}\|y\|_{n-1, \infty} \leq \\
\leq c_{n}\|A(\varepsilon)-A(0)\|_{n-1, \infty}\|y\|_{n, \infty} \quad \text { as } \quad \varepsilon \rightarrow 0+.
\end{gathered}
$$

Here, $c_{n}$ is a positive number independent of $y$. This number exists because $W_{\infty}^{n}$ is a Banach algebra. Hence,

$$
\|L(\varepsilon)-L(0)\| \leq c_{n}\|A(\varepsilon)-A(0)\|_{n-1, \infty} \rightarrow 0 \quad \text { as } \quad \varepsilon \rightarrow 0+,
$$

where $\|\cdot\|$ denotes the norm of a linear continuous operator on the pair of spaces

$$
L(\varepsilon):\left(W_{\infty}^{n}\right)^{m} \rightarrow\left(W_{\infty}^{n-1}\right)^{m} .
$$

The first implication is proved.

We now prove that the boundary condition (I) follows from condition $\left(a_{2}\right)$. Assume that condition $\left(a_{2}\right)$ is satisfied. Then

$$
Y^{\prime}+A(\varepsilon) Y=[L(\varepsilon) Y] \rightarrow[L(0) Y]=Y^{\prime}+A(0) Y \quad \text { in } \quad\left(W_{\infty}^{n-1}\right)^{m \times m}
$$

as $\varepsilon \rightarrow 0+$ for any matrix function $Y \in\left(W_{\infty}^{n}\right)^{m \times m}$. Moreover, the matrix function $[L(\varepsilon) Y]$ is formed by columns obtained are a result of the action of the operator $L(\varepsilon)$ on the corresponding columns of the matrix $Y$. Setting $Y(t) \equiv I_{m}$, we arrive at the required convergence of $A(\varepsilon) \rightarrow$ $A(0)$ in $\left(W_{\infty}^{n-1}\right)^{m \times m}$ as $\varepsilon \rightarrow 0+$. The second implication and, hence, Lemma 5 are proved.

Theorem 4 is proved.

\section{Proofs of Theorems 1 and 2}

Proof of Theorem 1. The sufficiency of conditions (0), (I), and (II) under which problem (1), (2) satisfies Definition 1 is established in Theorem 3. We prove the necessity. Assume that problem (1), (2) satisfies Definition 1. Then condition (0) is valid. It remains to show that this problem satisfies conditions (I) and (II). We split the proof into three steps: 
Step 1. We prove that the boundary-value problem (1), (2) satisfies the boundary condition (I). Under the condition $(*)$ of Definition 1, the operator

$$
(L(\varepsilon), B(\varepsilon)):\left(W_{\infty}^{n}\right)^{m} \rightarrow\left(W_{\infty}^{n-1}\right)^{m} \times \mathbb{C}^{m}
$$

is invertible for any $\varepsilon \in\left[0, \varepsilon_{1}\right)$. For any $\varepsilon \in\left[0, \varepsilon_{1}\right)$, we consider a matrix boundary-value problem

$$
\begin{aligned}
Y^{\prime}(t ; \varepsilon)+A(t ; \varepsilon) Y(t ; \varepsilon) & =O_{m}, \quad t \in(a, b), \\
{[B Y(\cdot ; \varepsilon)] } & =I_{m} .
\end{aligned}
$$

This boundary-value problem is a collection of $m$ boundary-value problems (1), (21) with righthand sides independent of $\varepsilon$. By assumption, this problem is uniquely solvable and its solution $Y(\cdot ; \varepsilon) \in\left(W_{\infty}^{n}\right)^{m \times m}$ satisfies the condition $Y(\cdot ; \varepsilon) \rightarrow Y(\cdot ; 0)$ in the space $\left(W_{\infty}^{n}\right)^{m \times m}$ as $\varepsilon \rightarrow 0+$. Note that $\operatorname{det} Y(t ; \varepsilon) \neq 0$ for any $t \in(a, b)$ because otherwise the columns of the matrix function $Y(\cdot ; \varepsilon)$ are linearly dependent, which contradicts the condition $[B Y(\cdot ; \varepsilon)]=I_{m}$. Hence,

$$
A(\cdot ; \varepsilon)=-Y^{\prime}(\cdot ; \varepsilon)(Y(\cdot ; \varepsilon))^{-1} \rightarrow-Y^{\prime}(\cdot ; 0)(Y(\cdot ; 0))^{-1}=A(\cdot ; 0)
$$

in the space $\left(W_{\infty}^{n}\right)^{m \times m}$ as $\varepsilon \rightarrow 0+$, i.e., condition (I) is satisfied.

Step 2. We now show that condition (II) is satisfied. First, we prove that $\|B(\varepsilon)\|=O(1)$ as $\varepsilon \rightarrow 0+$, where $\|\cdot\|$ is the norm of the bounded operator $B(\varepsilon):\left(W_{\infty}^{n}\right)^{m} \rightarrow \mathbb{C}^{m}$. Assume the contrary, i.e., that there exists a number sequence $\left(\varepsilon^{(k)}\right)_{k=1}^{\infty} \subset\left(0, \varepsilon_{1}\right)$ such that $\varepsilon^{(k)} \rightarrow 0$ and

$$
0<\left\|B\left(\varepsilon^{(k)}\right)\right\| \rightarrow \infty, \quad \varepsilon \rightarrow 0+.
$$

For each number $k$, we choose a vector function $x_{k} \in\left(W_{\infty}^{n}\right)^{m}$ such that

$$
\left\|x_{k}\right\|_{n, \infty}=1 \quad \text { and } \quad\left\|B\left(\varepsilon^{(k)}\right) x_{k}\right\|_{\mathbb{C}^{m}} \geq \frac{1}{2}\left\|B\left(\varepsilon^{(k)}\right)\right\| .
$$

We set

$$
\begin{gathered}
y\left(\cdot ; \varepsilon^{(k)}\right):=\left\|B\left(\varepsilon^{(k)}\right)\right\|^{-1} x_{k} \\
f\left(\cdot ; \varepsilon^{(k)}\right):=L\left(\varepsilon^{(k)}\right) y\left(\cdot ; \varepsilon^{(k)}\right) \\
c\left(\varepsilon^{(k)}\right):=B\left(\varepsilon^{(k)}\right) y\left(\cdot ; \varepsilon^{(k)}\right) .
\end{gathered}
$$

Since $y\left(\cdot ; \varepsilon^{(k)}\right) \rightarrow 0$ in the space $\left(W_{\infty}^{n}\right)^{m}$ as $\varepsilon \rightarrow 0+$, we have $f\left(\cdot ; \varepsilon^{(k)}\right) \rightarrow 0$ in $\left(W_{\infty}^{n-1}\right)^{m}$ because it has already been shown that $A(\cdot ; \varepsilon)$ satisfies condition (I). Since the finite-dimensional space $\mathbb{C}^{m}$ is locally compact, the inequalities

$$
1 / 2 \leq\left\|c\left(\varepsilon^{(k)}\right)\right\|_{\mathbb{C}^{m}} \leq 1
$$

are true. Passing to a subsequence of numbers $\varepsilon^{(k)}$, we can assume that $c\left(\varepsilon^{(k)}\right) \rightarrow c(0)$ as $k \rightarrow \infty$, where $c(0)$ is a nonzero vector in $\mathbb{C}^{m}$. Thus, for any number $k$, the vector function $y\left(\cdot ; \varepsilon^{(k)}\right) \in\left(W_{\infty}^{n}\right)^{m}$ is a unique solution of the boundary-value problem

$$
\begin{gathered}
L\left(\varepsilon^{(k)}\right) y\left(t ; \varepsilon^{(k)}\right)=f\left(t ; \varepsilon^{(k)}\right), \quad t \in(a, b), \\
B\left(\varepsilon^{(k)}\right) y\left(\cdot ; \varepsilon^{(k)}\right)=c\left(\varepsilon^{(k)}\right) .
\end{gathered}
$$

Recall that $f\left(\cdot ; \varepsilon^{(k)}\right) \rightarrow 0$ in $\left(W_{\infty}^{n-1}\right)^{m}$ and $c\left(\varepsilon^{(k)}\right) \rightarrow c(0) \neq 0$ in $\mathbb{C}^{m}$ as $k \rightarrow \infty$. Under the condition $(* *)$ of Definition 1, the function $y\left(\cdot ; \varepsilon^{(k)}\right)$ converges in the space $\left(W_{\infty}^{n}\right)^{m}$ to the 
unique solution $y(\cdot ; 0)$ of the limit boundary-value problem formed by the differential equation $L(0) y(t, 0)=0, t \in(a, b)$, and the inhomogeneous boundary condition $B(0) y(\cdot ; 0)=c(0)$. Since $y\left(\cdot ; \varepsilon^{(k)}\right) \rightarrow 0$ in the same space, we conclude that $y(\cdot ; 0) \equiv 0$, which contradicts the boundary condition. Therefore, this assumption is not true, i.e., $\|B(\varepsilon)\|=O(1)$ as $\varepsilon \rightarrow 0+$.

Step 3. We now show that condition (II) is satisfied. It follows from the result established above that there exist numbers $\gamma^{\prime}>0$ and $\varepsilon^{\prime} \in\left(0, \varepsilon_{1}\right)$ such that $\|(L(\varepsilon), B(\varepsilon))\| \leq \gamma^{\prime}$ for all $\varepsilon \in\left[0, \varepsilon^{\prime}\right)$, where $\|\cdot\|$ is the norm of a bounded operator acting from the space $\left(W_{\infty}^{n}\right)^{m}$ into the space $\left(W_{\infty}^{n-1}\right)^{m} \times \mathbb{C}^{m}$. We arbitrarily choose a vector function $y \in\left(W_{\infty}^{n}\right)^{m}$ and set $f(\cdot ; \varepsilon):=L(\varepsilon) y$ and $c(\varepsilon):=B(\varepsilon) y$ for any $\varepsilon \in\left[0, \varepsilon_{0}\right)$. Hence, in view of condition (**), as $\varepsilon \rightarrow 0+$, we get

$$
\begin{gathered}
\|B(\varepsilon) y-B(0) y\|_{\mathbb{C}^{m}} \leq\|(f(\cdot ; \varepsilon), c(\varepsilon))-(f(\cdot ; 0), c(0))\|_{\left(W_{\infty}^{n-1}\right)^{m} \times \mathbb{C}^{m}}= \\
=\left\|(L(\varepsilon), B(\varepsilon))(L(\varepsilon), B(\varepsilon))^{-1}(f(\cdot ; \varepsilon), c(\varepsilon))-(f(\cdot ; 0), c(0))\right\|_{\left(W_{\infty}^{n-1}\right)^{m} \times \mathbb{C}^{m}} \leq \\
\leq \gamma^{\prime}\left\|(L(\varepsilon), B(\varepsilon))^{-1}((f(\cdot ; \varepsilon), c(\varepsilon))-(f(\cdot ; 0), c(0)))\right\|_{n, \infty}= \\
=\gamma^{\prime}\left\|(L(0), B(0))^{-1}(f(\cdot ; 0), c(0))-(L(\varepsilon), B(\varepsilon))^{-1}(f(\cdot ; 0), c(0))\right\|_{n, \infty} \rightarrow 0 .
\end{gathered}
$$

Since

$$
\|B(\varepsilon)\|=O(1) \quad \text { and } \quad\|B(\varepsilon) y-B(0) y\|_{\mathbb{C}^{m}} \rightarrow 0,
$$

we conclude that $B(\varepsilon) y$ converges to $B(0) y$ in $\mathbb{C}^{m}$ for any $y \in\left(W_{\infty}^{n}\right)^{m}$. Thus, the boundaryvalue problem (1), (2) satisfies condition (II).

Proof of Theorem 2. First, we show that the left-hand side of the two-sided inequality (44) holds. We set

$$
f(\cdot, \varepsilon):=L(\varepsilon) y(\cdot ; \varepsilon), \quad c(\varepsilon):=B(\varepsilon) y(\cdot ; \varepsilon) .
$$

The strong convergence of inverse operators

$$
(L(\varepsilon), B(\varepsilon)) \stackrel{s}{\rightarrow}(L(0), B(0)), \quad \varepsilon \rightarrow 0+,
$$

follows from the boundary conditions (I) and (II). Hence, there exist numbers $\gamma^{\prime}>0$ and $\varepsilon \in\left(0, \varepsilon_{2}^{\prime}\right)$ such that the norm of this operator satisfies the inequality

$$
\|(L(\varepsilon), B(\varepsilon))\| \leqslant \gamma^{\prime} .
$$

Indeed, if we assume the contrary, then we can find a sequence of positive numbers $\left(\varepsilon^{(k)}\right)_{k=1}^{\infty}$ such that

$$
\varepsilon^{(k)} \rightarrow 0 \quad \text { and } \quad\left\|\left(L\left(\varepsilon^{(k)}\right), B\left(\varepsilon^{(k)}\right)\right)\right\| \rightarrow \infty \quad \text { as } \quad k \rightarrow \infty .
$$

However, by the Banach-Steinhaus theorem, this contradicts the fact that $\left(L\left(\varepsilon^{(k)}\right), B\left(\varepsilon^{(k)}\right)\right)$ strongly converge to $(L(0), B(0))$ as $k \rightarrow \infty$. By using (26) and (27), for any $\varepsilon \in\left(0, \varepsilon_{2}^{\prime}\right)$, we conclude that

$$
\begin{gathered}
\|L(\varepsilon) y(\cdot ; 0)-f(\cdot ; \varepsilon)\|_{n-1, \infty}+\|B(\varepsilon) y(\cdot ; 0)-c(\varepsilon)\|_{\mathbb{C}^{m}}= \\
=\|L(\varepsilon) y(\cdot ; 0)-L(\varepsilon) y(\cdot ; \varepsilon)\|_{n-1, \infty}+\|B(\varepsilon) y(\cdot ; 0)-B(\varepsilon) y(\cdot ; \varepsilon)\|_{\mathbb{C}^{m}} \leq \\
\leq\|L(\varepsilon)\|\|y(\cdot ; 0)-y(\cdot ; \varepsilon)\|_{n, \infty}+\|B(\varepsilon)\|\|y(\cdot ; 0)-y(\cdot ; \varepsilon)\|_{n, \infty} \leq \gamma^{\prime}\|y(\cdot ; 0)-y(\cdot ; \varepsilon)\|_{n, \infty} .
\end{gathered}
$$

Thus, we have established the left-hand side of inequality (41) with $\gamma_{1}:=1 / \gamma^{\prime}$. 
We now prove the right-hand side of the two-sided inequality (41). By Theorem 1, the operator $(L(\varepsilon), B(\varepsilon))$ has a bounded inverse operator $(L(\varepsilon), B(\varepsilon))^{-1}$ for any $\varepsilon \in\left(0, \varepsilon_{2}^{\prime}\right)$. Moreover, we have the following strong convergence:

$$
(L(\varepsilon), B(\varepsilon))^{-1} \stackrel{s}{\rightarrow}(L(0), B(0))^{-1}, \quad \varepsilon \rightarrow 0+.
$$

Indeed, for any $f \in\left(W_{\infty}^{n-1}\right)^{m}$ and $c \in \mathbb{C}^{m}$, under the condition $(* *)$ of Definition 1, we get the following convergence:

$$
(L(\varepsilon), B(\varepsilon))^{-1}(f ; c)=: y(\cdot ; \varepsilon) \rightarrow y(\cdot ; 0):=(L(0), B(0))^{-1}(f ; c)
$$

in $\left(W_{\infty}^{n}\right)^{m}$ as $\varepsilon \rightarrow 0+$. As above, by the Banach-Steinhaus theorem, the norms of these inverse operators are bounded, i.e., there exist positive numbers $\varepsilon_{2}$ and $\gamma_{2}$ such that the norm of the inverse operator

$$
\left\|(L(\varepsilon), B(\varepsilon))^{-1}\right\| \leqslant \gamma_{2} .
$$

Thus, for any $\varepsilon \in\left(0, \varepsilon_{2}\right)$, the relations

$$
\begin{gathered}
\|y(\cdot ; 0)-y(\cdot ; \varepsilon)\|_{n, \infty}=\left\|(L(\varepsilon), B(\varepsilon))^{-1}(L(\varepsilon), B(\varepsilon))(y(\cdot ; 0)-y(\cdot ; \varepsilon))\right\|_{n, \infty} \leq \\
\leq \gamma_{2}\left(\|L(\varepsilon) y(\cdot ; 0)-f(\cdot ; \varepsilon)\|_{n-1, \infty}+\|B(\varepsilon) y(\cdot ; 0)-c(\varepsilon)\|_{\mathbb{C}^{m}}\right)
\end{gathered}
$$

are true. This directly yields the right-hand side of the two-sided estimate (41).

\section{References}

[1] A. A. Boichuk and A. M. Samoilenko, Generalized Inverse Operators and Fredholm Boundary-Value Problems, VSP, ZeistBoston (2004).

[2] I. T. Kiguradze, Some Singular Boundary-Value Problems for Ordinary Differential Equations [in Russian], Tbilisi University, Tbilisi (1975).

[3] I. T. Kiguradze, "Boundary-value problems for systems of ordinary differential equations, " in: VINITI [in Russian], 30 (1987), pp. 3-103.

[4] T. I. Kodlyuk, V. A. Mikhailets, and N. V. Reva, "Limit theorems for one-dimensional boundary-value problems, " Ukr. Mat. Zh., 65, No. 1, 70-81 (2013); English translation: Ukr. Math. J., 65, No. 1, 77-90 (2013).

[5] V. A. Mikhailets, O. B. Pelekhata, and N. V. Reva, "Limit theorems for one-dimensional boundary-value problems, " Ukr. Mat. Zh., 65, No. 1, 70-81 (2013); English translation: Ukr. Math. J., 65, No. 1, 77-90 (2013).

[6] V. A. Mikhailets and G. A. Chekhanova, "Limit theorem for general one-dimensional boundary-value problems, " J. Math. Sci., 204, No. 3, 333-342 (2015).

[7] E. V. Gnyp, T. I. Kodlyuk, and V. A. Mikhailets, "Fredholm boundary-value problems with parameter in Sobolev spaces," Ukr. Mat. Zh., 67, No. 5, 584-591 (2015); English translation: Ukr. Math. J., 67, No. 5, 658-667 (2015).

[8] T. I. Kodlyuk and V. A. Mikhailets, "Solutions of one-dimensional boundary-value problems with a parameter in Sobolev spaces," J. Math. Sci., 190, No. 4, 589-599 (2013). 
[9] Y. V. Hnyp, V. A. Mikhailets, and A. A. Murach, "Parameter-dependent one-dimensional boundary-value problems in Sobolev spaces, "Electron. J. Different. Equat., No. 81 (2017).

[10] V. A. Mikhailets, A. A. Murach, and V. O. Soldatov, "Continuity in a parameter of solutions to generic boundary-value problems, "Electron. J. Qual. Theory Different. Equat., No. 87 (2016).

[11] O. M. Atlasiuk and V. A. Mikhailets, "Fredholm one-dimensional boundary-value problems in Sobolev spaces, " Ukr. Mat. Zh., 70, No. 10, 1324-1333 (2018). 